\title{
Stent graft treatment for ruptured pseudoaneurysms of the splanchnic arteries after pancreaticoduodenectomy: a case report
}

\author{
Masaru Nemoto ${ }^{1 *}$, Ken Koyama ${ }^{2}$, Midori Enokido ${ }^{2}$, Shohei Kudo ${ }^{3}$ and Fuyo Yoshimi ${ }^{1}$
}

\begin{abstract}
Background: Hemorrhage due to a ruptured splanchnic pseudoaneurysm followed by the formation of a postoperative pancreatic fistula is the most severe complication of a pancreatectomy, sometimes leading to a fatal outcome. Stent graft placement to control the hemorrhage due to the pseudoaneurysm is a validated treatment option, but once the stent graft is infected, infection control is complicated. We report a case of a ruptured pseudoaneurysm of the splanchnic artery after pancreaticoduodenectomy to evaluate the stent graft treatment.

Case presentation: A 77-year-old man underwent pylorus-preserving pancreaticoduodenectomy for suspected distal bile duct cancer. Hemorrhage from a pseudoaneurysm of the common hepatic artery due to the formation of the pancreatic fistula was detected on postoperative day 9, and a stent graft was successfully placed with the preservation of hepatic arterial blood flow. On postoperative day 12, new-onset hemorrhage from a pseudoaneurysm of the right hepatic artery developed, and a stent graft was similarly placed, but immediately occluded. Refractory pancreatic and biliary fistulas developed and required continuous drainage. On postoperative day 85, computed tomography revealed the presence of air within the latter stent graft, which indicated infection of the stent graft. The patient died due to sepsis caused by the graft infection.

Conclusion: Stent graft placement for the treatment of hemorrhage of a pseudoaneurysm secondary to a postoperative pancreatic fistula, following pancreaticoduodenectomy, is an effective treatment option as it achieves immediate hemostasis and maintains end-organ perfusion. However, stent graft infection is the most detrimental complication.
\end{abstract}

Keywords: Stent graft, Pancreaticoduodenectomy, Pseudoaneurysm

\section{Background}

Pancreaticoduodenectomy (PD) is a standard surgical procedure for the treatment of pancreatic head and distal bile duct disease. The mortality rate due to a PD has decreased to less than $5 \%$, but the morbidity rate remains high, ranging between 30 and $50 \%$ [1]. Hemorrhage and postoperative pancreatic fistula (POPF)

\footnotetext{
* Correspondence: mmmnemoto@gmail.com

${ }^{1}$ Department of Surgery, Ibaraki Prefectural Central Hospital \& Cancer Center, 6528 Koibuchi, Kasama, Ibaraki 309-1793, Japan

Full list of author information is available at the end of the article
}

formation are the most severe complications following PD, sometimes leading to a fatal outcome. Delayed hemorrhage of ruptured splanchnic pseudoaneurysms caused by a POPF, which mainly occurs in the common hepatic artery (CHA) and the stump of the gastroduodenal artery (GDA), is a life-threatening condition with a high mortality rate and requires immediate intervention [2]. Surgical hemostasis and transcatheter arterial embolization (TAE) are the treatment options for the hemorrhage of pseudoaneurysms. Alternatively, treatment using stent graft placement has been sporadically 
reported and results in excellent clinical outcomes with the preservation of end-organ arterial blood flow [3]. Herein we report a case of ruptured splanchnic pseudoaneurysms secondary to a POPF following PD, which was treated with the placement of a stent graft to obtain primary hemostasis, but the stent graft was infected and lead to a fatal outcome.

\section{Case presentation}

A 77-year-old man, with a background of hypertension and diabetes mellitus, underwent pylorus-preserving PD for suspected distal bile duct cancer. He developed a pancreatic anastomotic leakage on postoperative day (POD) 6, which was draining from a drain placed at the pancreaticojejunal anastomosis during surgery. Sentinel bleeding from the drain was noted on POD 8, and an immediate contrast-enhanced computed tomography (CT) scan revealed no obvious bleeding points. However, a massive hemorrhage from the drain suddenly developed on POD 9, and emergency angiography detected a pseudoaneurysm at the CHA, which had caused the bleeding (Fig. 1a). The right hepatic artery (RHA) independently branched from the origin of the CHA. Based on the general condition of the patient and the likelihood of postoperative adhesions, the placement of a stent graft was the treatment of choice. A 6-F sheath was passed through the celiac trunk via the femoral artery. A $6 \mathrm{~mm} \times 5 \mathrm{~cm}$ Viabahn stent (W.L. Gore, Flagstaff, Ariz) was placed from the CHA and the distal part of the replaced RHA to the left hepatic artery (LHA) with heparinization (Fig. 1b). Angiography following stent graft placement revealed the absence of a pseudoaneurysm and preserved hepatic arterial blood flow. His vital signs were stable after the intervention, and antiplatelet therapy was initiated using aspirin $100 \mathrm{mg} /$ day. However, re-bleeding from the drain appeared on POD 12. Angiography was immediately performed, and contrast extravasation from an aneurysm at the RHA was detected (Fig. 1c). A $5 \mathrm{~mm} \times 5 \mathrm{~cm}$ Viabahn stent was placed into the RHA with heparinization. Subsequent angiography showed the occlusion of the latter Viabahn stent, while the patency of the former Viabahn stent was maintained (Fig. 1d). Due to sufficient hepatic arterial blood flow through the RHA, by retrograde flow via the LHA, no additional intervention for the stent graft occlusion was performed, and the patient was carefully observed. A biliary fistula appeared on POD 15, which might be caused by the occlusion of RHA and

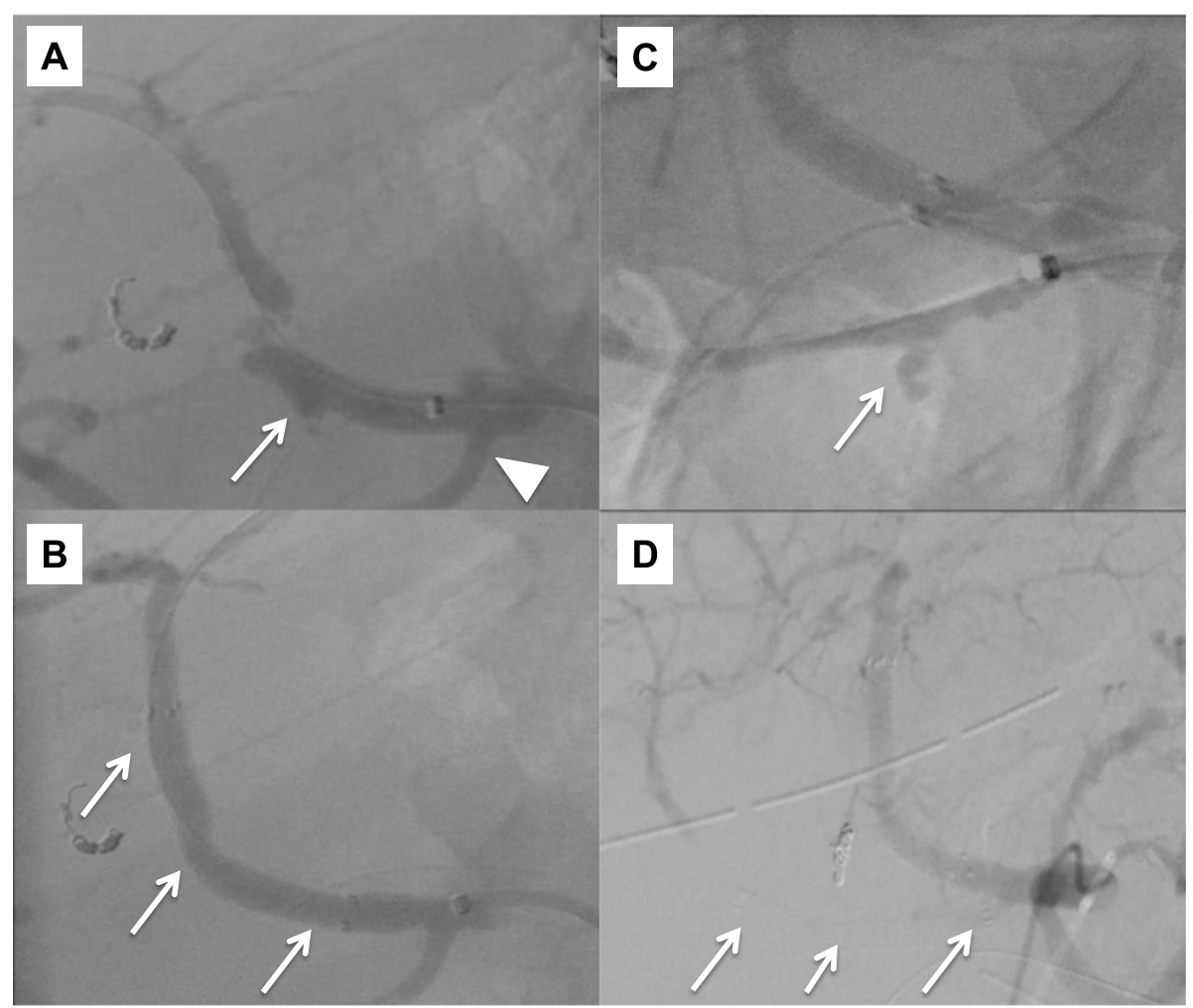

Fig. 1 Pre- and postoperative angiogram. a Angiogram reveals contrast extravasation from the pseudoaneurysm at the CHA (arrow). The RHA is branched independently from the CHA (arrowhead). b A stent graft, $6 \mathrm{~mm}$ in diameter and $5 \mathrm{~cm}$ in length, is placed from the CHA and distal part of the replaced RHA to the LHA (arrows). c Angiogram shows contrast extravasation from the RHA (arrow). $\mathbf{d}$ A stent graft, $5 \mathrm{~mm}$ in diameter and $5 \mathrm{~cm}$ in length, was placed in the RHA, but occluded soon after (arrows) 
compression of massive hematoma around the biliary anastomosis. An abdominal abscess around the biliary anastomosis was detected, and percutaneous CT-guided drainage was performed on POD 28, and antibiotic therapy based on a bacterial culture was initiated. These pancreatic and biliary fistulas were considered to be a refractory complication. On POD 85, the patient's general condition worsened, and the CT scan revealed the presence of air within the latter occluded stent graft, which indicated that the stent graft had become infected (Fig. 2). Escherichia coli was confirmed by blood culture, and the patient died on POD 91 due to sepsis caused by the graft infection. This study was approved by the ethics committee of our institution, and informed consent was obtained from the patient's next-of-kin.

\section{Discussion}

Pseudoaneurysms are believed to be caused by anatomical leakages, mainly due to pancreatic fistulas or injury of the arterial wall during operative dissection and lymphadenectomy [4]. It is crucial to detect and initiate interventions in cases of hemorrhaging due to pseudoaneurysms in order to improve outcomes. In this case, we presumed that the local infection secondary to the POPF had caused the pseudoaneurysms in the CHA and RHA to develop.

Despite the identification of the sentinel bleeding and having performed the CT scan in our case, the pseudoaneurysms were not clearly detected, and angiography was canceled. Sentinel bleeding is considered a sign of pseudoaneurysm bleeding prior to massive hemorrhage and is often observed in $45-78 \%$ of patients who have undergone PD [5]. Angiography has been recommended if this critical sign is identified [6].

Surgical re-exploration is the conventional management of hemorrhage due to a pseudoaneurysm.

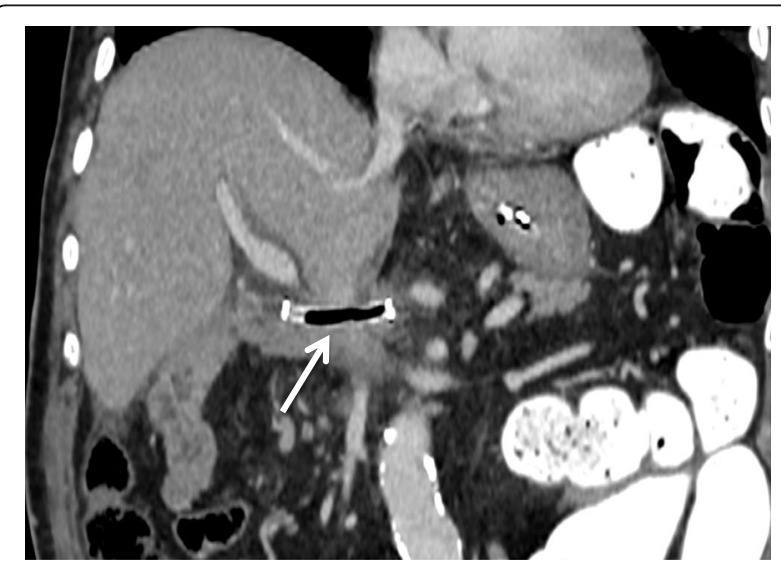

Fig. 2 Enhanced CT on POD 85. CT reveals the presence of air within the occluded stent graft, which indicates stent graft infection (arrow)
However, the surgery is difficult to perform due to tissue fragility and postoperative adhesions, which results in a significant risk of mortality and morbidity. The operative mortality rate has been reported as $43-50 \%$. Therefore, surgical re-exploration is recommended when an endovascular approach is difficult [7]. Endovascular treatments, including TAE and stent graft placement, have been accepted as alternative treatment options for postpancreatectomy hemorrhage. The advantages of endovascular treatments are the ability to perform these treatments percutaneously and immediately without the need for general anesthesia. TAE has been performed with high success and a lower mortality and morbidity rate when compared to open surgery [8]. However, liver abscess formation and liver dysfunction due to endorgan ischemia and re-bleeding may occur, resulting in an increasing rate of mortality [8].

To avoid these complications, stent graft placement is preferred over TAE. Stent graft placement provides excellent flexibility, enables precise deployment of the stent, and maintains end-organ arterial blood flow. Several reports on stent graft placement for the treatment of pseudoaneurysms after PD have recently been published $[9,10]$. Asai et al. reviewed 34 cases of stent graft placement for visceral delayed arterial hemorrhage after pancreatic-head resection, and the hemorrhage site was found to be the hepatic artery in 27 patients, the superior mesenteric artery (SMA) in 5 patients, and the splenic artery (SA) in 2 patients [11]. The technical success rate was $100 \%$. The complications following stent graft placement included the development of a liver abscess (2.9\%), re-bleeding (11.8\%), and stent occlusion (5.9\%). It has been recommended that double antiplatelet treatment (DAPT) be administered after stent graft placement in the visceral artery, for the prevention of stent graft occlusion. However, when hemorrhaging occurs, it is difficult to initiate the DAPT, and single antiplatelet treatment might be preferred. The morbidity associated with stent graft placement has been considered the lowest when compared to that in TAE and re-laparotomy. However, stent graft infection is the most detrimental complication [8]. Four cases with the development of sepsis following stent graft placement for visceral hemorrhage after PD have been reported. Only 1 case of stent graft infection following placement for a pseudoaneurysm of the SMA has been reported [3]. In this case, air was present in the occluded stent graft due to abscess formation around the SMA with an unhealed POPF 4 months after stent graft placement. The patient died due to sepsis 5 months after that. Three cases have been reported in which 2 patients have died due to intra-abdominal sepsis, and 1 patient had died due to an unknown cause (Table 1) [12-14]. It is presumed that the occlusion of the stent graft might be a risk factor for the development of an infection as the thrombus within the stent graft might act as a medium for bacterial growth. 
Table 1 Cases of stent graft infection or sepsis following stent graft placement for visceral hemorrhage after pancreaticoduodenectomy

\begin{tabular}{llllllll}
\hline Author & Year & Age & Sex & Bleeding point & Stent type & Outcome & Cause \\
\hline Stoupis et al. [12] & 2006 & 60 & Male & CHA & Jostent graft & Died after 10 days & Sepsis (intra-abdominal sepsis) \\
Heiss et al. [13] & 2007 & 72 & Male & SA & Advanta & Died after 3 months & Sepsis (unknown) \\
Suzuki et al. [3] & 2009 & 70 & Female & SMA & Passager & Died after 5 months & Stent graft infection and sepsis \\
Wang et al. [14] & 2010 & 53 & Male & CHA & Jostent graft & Died after 12 days & Sepsis (intra-abdominal sepsis) \\
Present case & 2019 & 77 & Male & CHA, RHA & Viabahn & Died after 91 days & Stent graft infection and sepsis \\
\hline
\end{tabular}

CHA common hepatic artery, SA splenic artery, SMA superior mesenteric artery, RHA right hepatic artery

Uncontrolled local infection with a POPF or a biliary fistula is another risk factor for stent graft infection. The risk of infection is considered similar in TAEs and stent grafts [12]. In our case, the placement of the stent graft was selected based on the general condition of the patient and the anatomical location of the pseudoaneurysm. Although percutaneous drainage of the POPF and the biliary fistula was performed in order to control local infection, the contact of abscess formed by the refractory fistula development to the occluded stent graft could have caused stent graft infection. Limitations of this study include the retrospective study design and small sample size.

\section{Conclusions}

Stent graft placement for the treatment of hemorrhage of a pseudoaneurysm secondary to a POPF after PD is an effective treatment option. It immediately achieves hemostasis and maintains end-organ perfusion better than that in TAE and surgical re-exploration. If local infection around the pseudoaneurysm is uncontrolled, and the stent graft is occluded, a lack of immediate infection control may lead to a fatal outcome.

\section{Abbreviations}

PD: Pancreaticoduodenectomy; POPF: Postoperative pancreatic fistula; TAE: Transcatheter arterial embolization; CHA: Common hepatic artery; GDA: Gastroduodenal artery; RHA: Right hepatic artery; LHA: Left hepatic artery; POD: Postoperative day; SMA: Superior mesenteric artery; DAPT: Double antiplatelet treatment; SA: Splenic artery; CT: Computed tomography

\section{Acknowledgments}

We thank Editage (www.editage.jp) for providing the English language editing.

\section{Authors' contributions}

MN drafted the manuscript. KK, ME, SK, and FY participated in the design of this case presentation. All authors have read and approved the final manuscript.

\section{Funding}

This research did not receive any specific grant from funding agencies in the public, commercial, or not-for-profit sectors.

\section{Availability of data and materials}

All datasets supporting the conclusions of this article are included in this published article.

\section{Competing interest}

None
Ethics approval and consent to participate

This case report was approved by the Institutional Ethics Committee. Written informed consent was obtained from the patient.

\section{Consent for publication}

Informed consent was obtained from the patient for the publication of their data.

\section{Author details}

${ }^{1}$ Department of Surgery, Ibaraki Prefectural Central Hospital \& Cancer Center, 6528 Koibuchi, Kasama, Ibaraki 309-1793, Japan. ²Department of Diagnostic and interventional radiology, Ibaraki Prefectural Central Hospital \& Cancer Center, 6528 Koibuchi, Kasama, Ibaraki 309-1793, Japan. ${ }^{3}$ Department of Hepato-Biliary-Pancreatic Surgery, University of Tokyo, 7 Chome-3-1 Hongo, Bunkyo City, Tokyo 113-8654, Japan.

Received: 31 December 2019 Accepted: 24 May 2020

Published online: 01 June 2020

\section{References}

1. De Oliveira ML, Winter JM, Schafer M, Cunningham SC, Cameron JL, Yeo CJ, et al. Assessment of complications after pancreatic surgery: a novel grading system applied to 633 patients undergoing pancreaticoduodenectomy. Ann Surg. 2006;244:931-7.

2. van Berge Henegouwen MI, Allema JH, van Gulik TM, van Gulik TM, Verbeek PC, Obertop H, et al. Delayed massive haemorrhage after pancreatic and biliary surgery. Br J Surg. 1995:82:1527-31.

3. Suzuki K, Mori Y, Komada T, Matsushima M, Ota T, Naganawa S. Stent-graft treatment for bleeding superior mesenteric artery pseudoaneurysm after pancreaticoduodenectomy. Cardiovasc Intervent Radiol. 2009;32:762-6.

4. Rumstadt B, Schwab M, Korth P, Samman M, Trede M. Hemorrhage after pancreatoduodenectomy. Ann Surg. 1998;227:236-41.

5. Lee HG, Heo JS, Choi SH, Choi DW. Management of bleeding from pseudoaneurysms following pancreaticoduodenectomy. World J Gastroenterol. 2010;16:1239-44.

6. Tien YW, Wu YM, Liu KL, Ho CM, Lee PH. Angiography is indicated for every sentinel bleed after pancreaticoduodenectomy. Ann Surg Oncol. 2008;15: 1855-61.

7. Limongelli $\mathrm{P}$, Khorsandi SE, Pai M, Jackson JE, Tait $\mathrm{P}$, Tierris J, et al. Management of delayed postoperative hemorrhage after pancreaticoduodenectomy: a meta-analysis. Arch Surg. 2008;143:1001-7.

8. Ding X, Zhu J, Zhu M, Li C, Jian W, Jiang J, et al. Therapeutic management of hemorrhage from visceral artery pseudoaneurysms after pancreatic surgery. J Gastrointest Surg. 2011;15:1417-25.

9. Fujioka S, Suzuki F, Funamizu N, Okamoto T, Munakata K, Ashida H, et al. Stent graft placement and balloon dilation for pseudoaneurysm complicated by distal arterial stenosis of the hepatic artery after pancreaticoduodenectomy. Surg Case Rep. 2015;1:60.

10. You Y, Choi SH, Choi DW, Heo JS, Han IW, Han S, et al. Long-term clinical outcomes after endovascular management of ruptured pseudoaneurysm in patients undergoing pancreaticoduodenectomy. Ann Surg Treat Res. 2019; 96:237-49.

11. Asai K, Watanabe M, Kusachi S, Matsukiyo H, Saito T, Kodama H, et al. Successful treatment of a common hepatic artery pseudoaneurysm using a coronary covered stent following pancreatoduodenectomy: report of a case. Surg Today. 2014;44:160-5. 
12. Stoupis C, Ludwig K, Inderbitzin D, Do DD, Triller J. Stent grafting of acute hepatic artery bleeding following pancreatic head resection. Eur Radiol. 2007;17:401-8

13. Heiss P, Bachthaler M, Hamer OW, Piso P, Herold T, Schlitt HJ, et al. Delayed visceral arterial hemorrhage following Whipple's procedure: minimally invasive treatment with covered stents. Ann Surg Oncol. 2008;15:824-32.

14. Wang MQ, Liu FY, Duan F, Wang ZJ, Song P, Fan QS. Stent-grafts placement for treatment of massive hemorrhage from ruptured hepatic artery after pancreaticoduodenectomy. World J Gastroenterol. 2010;16:3716-22.

\section{Publisher's Note}

Springer Nature remains neutral with regard to jurisdictional claims in published maps and institutional affiliations.

\section{Submit your manuscript to a SpringerOpen ${ }^{\circ}$ journal and benefit from:}

- Convenient online submission

- Rigorous peer review

- Open access: articles freely available online

- High visibility within the field

- Retaining the copyright to your article

Submit your next manuscript at $\boldsymbol{\nabla}$ springeropen.com 